\title{
Robust PPG Peak Detection Using Dilated Convolutional
} Neural Networks

This paper was downloaded from TechRxiv (https://www.techrxiv.org).

\section{LICENSE}

CC BY 4.0

SUBMISSION DATE / POSTED DATE

28-08-2021 / 01-09-2021

\section{CITATION}

Kazemi, Kianoosh; Laitala, Juho; Azimi, Iman; Liljeberg, Pasi; Rahmani, Amir M. (2021): Robust PPG Peak Detection Using Dilated Convolutional Neural Networks. TechRxiv. Preprint. https://doi.org/10.36227/techrxiv.16529310.v1

$\mathrm{DOI}$ 


\title{
Robust PPG Peak Detection Using Dilated Convolutional Neural Networks
}

\author{
KIANOOSH KAZEMI, ${ }^{1}$ (Student Member, IEEE), JUHO LAITALA ${ }^{1}$, IMAN AZIMI ${ }^{1}$, \\ PASI LILJEBERG ${ }^{1}$, (Member, IEEE), AND AMIR M. RAHMANI ${ }^{2,3}$, (Senior Member, IEEE) \\ ${ }^{1}$ Department of Computing, University of Turku, FI-20014 Turku, Finland \\ ${ }^{2}$ Department of Computer Science, University of California at Irvine, Irvine, CA 92697, USA \\ ${ }^{3}$ School of Nursing, University of California at Irvine, Irvine, CA 92697, USA \\ Corresponding author: Kianoosh Kazemi ( kianoosh.k.kazemi@utu.fi).
}

This research received support from the Academy of Finland through the SLIM Project (grant numbers 316810 and 316811 ) and the U.S. National Science Foundation (NSF) through the UNITE Project (grant number SCC CNS-1831918.)

\begin{abstract}
Accurate peak determination from noise-corrupted photoplethysmogram (PPG) signal is the basis for further analysis of physiological quantities such as heart rate and heart rate variability. In the past decades, many methods have been proposed to provide reliable peak detection. These peak detection methods include rule-based algorithms, adaptive thresholds, and signal processing techniques. However, they are designed for noise-free PPG signals and are insufficient for PPG signals with low signal-to-noise ratio (SNR). This paper focuses on enhancing PPG noise-resiliency and proposes a robust peak detection algorithm for noise and motion artifact corrupted PPG signals. Our algorithm is based on Convolutional Neural Networks (CNN) with dilated convolutions. Using dilated convolutions provides a large receptive field, making our CNN model robust at time series processing. In this study, we use a dataset collected from wearable devices in health monitoring under free-living conditions. In addition, a data generator is developed for producing noisy PPG data used for training the network. The method performance is compared against other state-of-the-art methods and tested in SNRs ranging from 0 to $45 \mathrm{~dB}$. Our method obtains better accuracy in all the SNRs, compared with the existing adaptive threshold and transform-based methods. The proposed method shows an overall precision, recall, and F1-score $80 \%, 80 \%$, and $80 \%$ in all the SNR ranges. However, these figures for the other methods are below $78 \%, 77 \%$, and $77 \%$, respectively. The proposed method proves to be accurate for detecting PPG peaks even in the presence of noise.
\end{abstract}

INDEX TERMS Photoplethysmogram, Peak detection, Convolutional Neural Network, Wearable devices, Motion artifacts

\section{INTRODUCTION}

$\mathbf{T}$ HERE is a growing demand for ubiquitous health monitoring systems. These systems are developed to provide proactive healthcare solutions as well as reduce medical costs: e.g., providing efficiency and cost-savings for doctors, nurses, and pharmaceutical companies [1]. Fortunately, rapid advancement in the Internet of Things (IoT)-based systems and wearable devices offer opportunities for the development of health monitoring systems [2]. Such IoT-based healthcare systems can provide comprehensive patient care by leveraging various sensor types, communication units, and computing resources. Wearable electronics - such as wristbands and smart rings - enable the ubiquitous collection of biomedical signals, including electrocardiogram (ECG) and photoplethysmogram (PPG) [3].
PPG is a low-cost, non-invasive, and simple optical technique used for measuring the synchronous blood volume changes in tissue such as the surface of the finger, toe, wrist, and forehead [3]. This approach is widely used in wearable IoT-based applications due to its high level of feasibility and ease of measurement [4]. Collected PPG signals can be used to extract various health parameters, such as heart rate and heart rate variability. These health parameters are obtained by the determination of the systolic peaks in the PPG records. However, the quality of the PPG waveform is easily affected by surrounding noises such as background noises and motion artifacts. As users engage in a variety of physical activities, these noises are unavoidable in IoT-based healthcare systems. Subsequently, when the signal quality is poor (i.e., low signal-to-noise ratio (SNR)), accurate detection of peaks in 
PPG signals becomes challenging. This issue increases false peak determination, which results in inaccurate vital signs extraction.

Numerous studies have been proposed to determine the PPG signal peaks accurately. In some specified cases, the signal is inspected manually by experts, and then the location of the peaks is annotated. These methods are often implemented in hospitals and clinics and are mainly used as gold standard methods for validation [5]. However, implementation of such methods requires much time and domain knowledge, which is not feasible.

On the other hand, there are various automatic techniques employing different signal processing, filtering, and rulebased techniques. These methods mainly include adaptive threshold [6], transform-based techniques [7], derivative calculation [8], and computer-based filtering [9].

Adaptive threshold techniques are commonly used for peak detection. In these methods, a threshold is set based on the proceeding peaks amplitude, and the threshold constantly decays until it reaches the next peak. Then, the value of the threshold is updated, and the threshold is controlled by different features such as duration, amplitude, beat-to-beatintervals, and sampling frequency [10], [11]. Transformbased techniques are other alternatives for PPG peak detection. These methods mostly employ signal processing algorithms such as discrete wavelet transform [12], stationary wavelet transform [7] and Hilbert transform [13]. Waveletbased techniques are mostly used in preprocessing stage for denoising. These techniques decompose the signal into multiple subbands which have the same resolution as the original signal. Then, by composing the desire subbands, the informative part of the signal is regenerated, and the baseline wanders and high-frequency (HF) noises are eliminated. In contrast, Hilbert transform is utilized for envelope extraction and peak detection task. Hilbert transform is a powerful tool in analyzing the amplitude and frequency of a signal instantaneously. [14], and [13] indicate that the zero-crossing points in the Hilbert transform correspond to the peak locations. However, these methods are insufficient for noisecontaminated signals, and they become unreliable if the SNR drops below a certain level.

The conventional peak detection techniques in the literature are mainly designed for noise-free or low-noise PPG signals. Therefore, they are insufficient to determine PPG peaks' locations when the signal quality is poor due to motion artifacts and HF noises. These noises are inevitable in wearable-based health and well-being monitoring systems. We believe that a peak detection method is required to determine systolic peaks in noisy PPG, leveraging temporal information in the signal. The robustness of such a method requires to be investigated against different noise levels.

In this paper, we propose a Convolution Neural Networks (CNN)-based peak determination approach for PPG signals with different levels of motion artifacts. The convolution layers in our network are dilated, resulting in a high receptive field efficiency. Therefore, the network can use temporal in- formation in PPG peak detection and learn complex problems associated with the noisy PPG signals. Our analysis exploits PPG signals and motion artifacts collected by wearable devices in health monitoring under free-living conditions. We develop a generator function to produce PPG signals with a wide range of noise, augmenting the training data and creating noisy signals similar to real-life PPG records. Using the PPG signals, the proposed method is evaluated in comparison with state-of-the-art PPG peak detection methods. In summary, the major contributions of the paper are as follows:

- Proposing a dilated convolutional neural networks for addressing the problem of PPG peak detection in the presence of noise.

- Developing a generator function for producing PPG signals with different noise levels to be used for model training.

- Assessing the robustness of the proposed method using noisy PPG signals with SNRs ranging from 0 to $45 \mathrm{~dB}$.

- Evaluating the proposed method in terms of accuracy compared to conventional methods, including adaptive threshold and Hilbert transform.

- Providing the model implemented in Python for the community to be used in their solutions 1

The rest of the paper is organized as follows. The background and related work of this research is outlined in Section II and III. We introduce the dataset used in this work in Section IV. Section V describes the development of the proposed method in detail. In Section VI, we evaluate our method in comparison with other published methods. Finally, Section VIII concludes the paper.

\section{BACKGROUND}

In this section, we briefly describe PPG and artificial neural networks proposed for PPG-based applications.

\section{A. PHOTOPLETHYSMOGRAPHY}

PPG is a convenient method for sensing the blood flow rate at peripheral sites. Therefore, this signal can be used to determine the cardiac cycle [3]. The PPG sensor includes two main components: i.e., a light source and a photodetector. PPG signals are acquired by emitting light in different wavelengths (e.g., infrared, red, and green, often at 940, 660, and 550 nanometers, respectively) to the skin surface and capturing the reflected light via photodetectors. The infrared and red lights are commonly used for measuring heart rate and blood oxygen saturation. Furthermore, the green light is widely used in wearable devices such as smartwatches [15].

The variation in the PPG signal is associated with cardiac and respiration oscillations. Figure 1 indicates a view of a PPG signal, where the heart rate values can be estimated by measuring the difference of the time interval between two successive peaks. The signal consists of two main components: i.e., the alternating current (AC) and direct current

\footnotetext{
${ }_{1}^{1}$ https://github.com/HealthSciTech/Robust_PPG_PD
} 


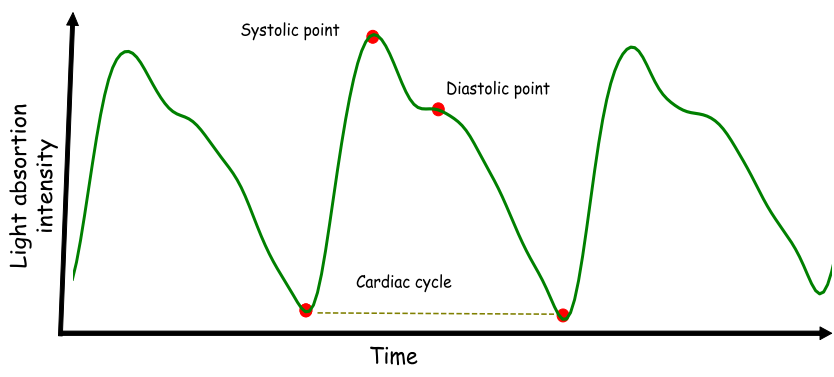

FIGURE 1. An example of a filtered PPG signal.

(DC). The AC part denotes synchronous cardiovascular fluctuations caused by cardiac activity, while the DC portion denotes various low-frequency elements of the blood flow, such as respiration [3], [16].

\section{B. ARTIFICIAL NEURAL NETWORKS}

Artificial neural networks are inspired by the human brain and imitate how biological neurons interact with one another, comprising an input layer, hidden layers, and an output layer [17]. Neural networks algorithms have been recently used in various PPG signal applications. In [18], a classification method based on multilayer perceptron (MLP) network was presented. In this study, an MLP network was trained to classify the pattern of the onset and systolic of the PPG signals with different window sizes. The preprocessing stage includes two steps: i.e., signals segmentation and smoothing using a simple mean square regression. Then, the results are fed to the network as features for pattern recognition. Reiss et al. [19] introduced a CNN architecture for heart rate estimation. In this study, the PPG signals and corresponding threeaxis accelerometer data were used to training the model. Naeini E. K. et al. [16] introduced a CNN architecture for real-time PPG quality assessment. They trained a classifier to discriminate between reliable and unreliable signals. This procedure was carried out by extracting the heart rate from PPG and simultaneously comparing the result with the ECG reference.

\section{RELATED WORK}

Several PPG peak detection methods with different complexities have been developed in the last decades. Most peak detection methods contain two main stages: i.e., 1) preprocessing (or filtering) and 2) envelope detection, and peak determination.

\section{1) Preprocessing}

Preprocessing is one of the significant stages in the PPG peak detection task. This step aims to remove the frequency component of the signal that does not reflect the fundamental features. In the preprocessing step, different filtering methods -such as low-pass filter, high-pass filter, singular value decomposition, and mode decomposition- are employed to suppress the baseline distortion and HF noises. Such methods enlarge the systolic peak part of the PPG signals. Tran et al. [20] proposed low-pass and high-pass filtering methods with cut-off frequencies of 0.4 and $8 \mathrm{~Hz}$ to remove the motion artifact and HF noise, respectively. In another study, [21], a low-pass filter with a cut-off frequency of $15 \mathrm{~Hz}$ was used for noise cancellation. A high-pass filter with a cut-off frequency of $0.01 \mathrm{~Hz}$ was also chosen to suppress the baseline wandering. Ricardo Ferro [13] used a digital fourth-order Chebyshev band-pass filter with the bandwidth of $0.5-16 \mathrm{~Hz}$ for eliminating the DC and HF components in the PPG signal. The major component of background noise is presented in the frequency range of 0.15 to $5.0 \mathrm{~Hz}$. This was achieved by employing a band-pass filtering method with cut-off frequencies of 0.5 and $5.5 \mathrm{~Hz}$ [22]. Prieto et al. [23] used a combination of two zero-phase delay fourthorder high-pass and eighth-order low-pass Butterworth filters with a bandwidth of $0.1-16 \mathrm{~Hz}$ to remove unwanted signals. Moving average filters were also utilized in noise surpassing. For instance, in [8], a 3-point bidirectional moving average was proposed to remove the phase delay caused by the filter.

In [24], a variational mode decomposition was used to enhance the signal quality and suppress the motion artifact. The decomposition was implemented in two stages to minimize the balancing error. Another method for noise removal is empirical mode decomposition which is computationally expensive. In this method, the signal is adaptively decomposed into intrinsic mode functions, and then by using averaging, the noise components are eliminated [9]. Paradkar et al. [7] introduced a singular value decomposition along with a moving average filter to extract the periodic component from the raw signal and reduce background noise.

\section{2) Envelope extraction and peak determination}

This stage generally includes extracting different features such as the maxima and minima, slope of the signal, and signals' envelope using well-known and robust algorithms, e.g., adaptive threshold and transform-based, to determine the PPG signal peaks.

The adaptive threshold is a common technique used for PPG peak detection. This method employs a constant specified by signals' temporal and frequency domain features and time intervals. The constant could be decaying or growing due to the dynamic nature of the PPG waveform [6], [20]. For example, Shin et al. [25] proposed to update the threshold according to different features such as the sampling frequency, preceding peaks, and standard deviation of the signal. In [10], the adaptive thresholding is equipped with a morphological filter to remove the low noises and use a slope sum function to pinpoint the location of peaks accurately. Van Gent [26], [27] presented a method based on an adaptive threshold followed by moving average and spline interpolation methods if the detected peaks show clipping. The complexity of the adaptive threshold PPG peak detection methods is low. However, the methods are sensitive to noise and fail to accurately 
identify the peaks when the PPG signal is contaminated by noise. In other words, the PPG signal changes rapidly due to noise, so the methods are incapable of selecting appropriate thresholds.

In addition, transform-based techniques are developed for PPG peak detection. These methods are mainly based on various non-linear transformations such as wavelet [7] and Hilbert [13] transforms, by which the signal's temporal and frequency domain features are extracted. Then, various thresholds -such as zero-crossing points or decision logicare set to extract the signals component and corresponding peaks in the original signals. In [13], a Hilbert transform was accompanied by moving average and Shannon energy envelope techniques to locate the position of the systolic peaks. Vadrevu et al. [28] introduced a stationary wavelet transform to extract two sets of coefficients from the PPG signal. Then, using multiscale sum and product, the peaks' sharpness was enhanced in the edges, and the other values remained near zero. Following that, the zero-crossing points were extracted to obtain the locations of the systolic peaks. Leveraging transform based-methods, the peak positions can be detected more accurately. For instance, Chakraborty et al. [29] proposed a robust algorithm -enabled by a Hilbert transform, amplitude thresholding, and signal derivativeto detect PPG systolic peaks. Their algorithm achieved a better performance in comparison to an adaptive threshold technique. However, the transform-based methods are still insufficient for wearable-based PPG, as they fail to precisely determine systolic peaks' in distorted PPG signals.

In another work, Jang et al. [11] introduced a positioning algorithm to locate PPG peaks. The method includes denoising and abnormal intervals removal steps. In [30], PPG peaks were automatically detected and corrected, exploiting a Poincare plot feature and envelope detection. The methods mentioned above are accurate when the PPG signal quality is good. However, they are highly susceptible to motion artifacts and environmental noises. They fail to differentiate false noisy peaks and systolic peaks and subsequently result in inaccurate peak detection. Moreover, the probability of false peak detection increase in signals with high heart rate. Consequently, these methods are insufficient for wearablebased monitoring, in which the users might engage in various physical activities.

\section{DATASET}

PPG dataset used in this paper is a part of a health monitoring study [31]. During the study, the participants were asked to wear wearable devices, by which their vital signs, physical activity, and sleep were tracked continuously. The monitoring was performed under free-living conditions, where the participants engaged in their normal daily routines.

The recruitment and data collection took place in southern Finland between July and August 2019. The recruitment started with the students and staff members of the University of Turku. More recruiting was then done with snowball sampling, and in the end, 46 individuals were recruited. All of
TABLE 1. Background information of the participants

\begin{tabular}{|c|c|c|}
\hline characteristic & Type & values \\
\hline \multirow{2}{*}{ Age (years), mean (SD) } & Men & $33.5(6.5)$ \\
\hline & Women & $31(6.8)$ \\
\hline \multirow{2}{*}{ BMI, mean (SD) } & Men & $25.58(2.94)$ \\
\hline & Women & $24.32(6.17)$ \\
\hline \multirow{3}{*}{ Exercise } & Almost daily & 9 \\
\hline & A few times a week & 19 \\
\hline & Once a week or fewer & 7 \\
\hline \multirow{4}{*}{ Education } & Primary school & 1 \\
\hline & High school & 7 \\
\hline & College & 8 \\
\hline & University & 20 \\
\hline \multirow{4}{*}{ Employment status } & Working & 27 \\
\hline & Unemployed & 1 \\
\hline & Student & 6 \\
\hline & Other & 1 \\
\hline
\end{tabular}

the participants were healthy individuals, and both males and females were present in equal numbers. Following exclusion criteria were used in the recruitment: (1) any restrictions using wearable devices at work, (2) restrictions regarding physical activity, (3) a diagnosed cardiovascular disease, and (4) symptoms of illness at recruitment time. Due to technical and practical issues, PPG signals from all 46 participants were not available, and data from 10 participants had to be excluded. Thus, PPG data from 36 participants were used in our analysis. Table 1 summarizes the background information of the participants.

All PPG signals were recorded with Samsung Gear Sport smartwatches [32]. The smartwatch has compact dimensions of $44.6 \times 42.9 \times 11.6 \mathrm{~mm}$, and it weighs 67 grams with the strap. The smartwatch is waterproof, its battery lasts about 3 days, and includes a PPG sensor and a built-in inertial measurement unit. The device runs an open-source Tizen operating system, enabling customized data collection and data transmission.

For the data collection, the participants were asked to wear the smartwatches on their non-dominant hands. The watches were programmed to collect data continuously for 24 hours at the sampling frequency of $20 \mathrm{~Hz}$. For the analysis, we upsample the PPG signals to $100 \mathrm{~Hz}$. The participants were also asked to send the collected data via Wi-Fi to our server using our Tizen app [31]. Our monitoring system is depicted in Figure 2, including the Samsung Gear sport smartwatch for data collection, a smartphone as a gateway layer for data transmission, and the cloud server.

This study was conducted following the ethical principles set by the Declaration of Helsinki and the Finnish Medical 


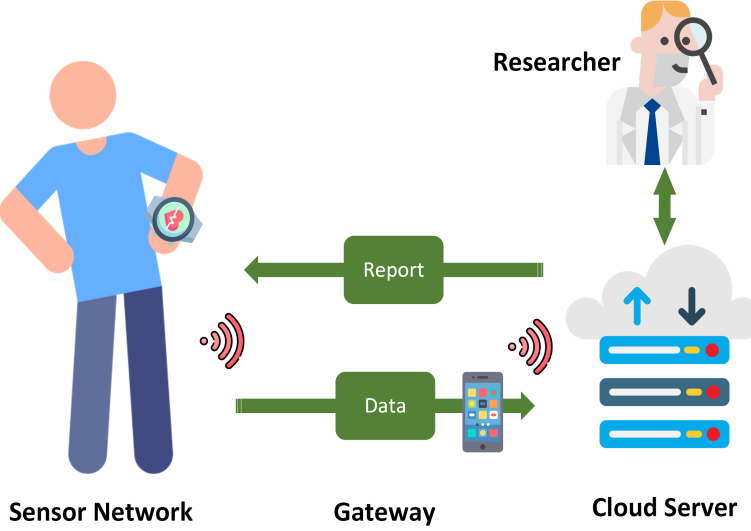

FIGURE 2. The monitoring system used for PPG collection.

Research Act (No 488/1999). In addition, the University of Turku Ethics committee for Human Sciences gave a favorable statement (No 44/2019) of the study protocol. All study participants received both oral and written information about the study before their written consent was obtained. Study participation was entirely voluntary, and at all times, the participant had a right to withdraw from the study without giving any reason. At the end of the monitoring period, each participant was compensated with a $20 €$ gift card.

\section{DEEP LEARNING-BASED PPG PEAK DETECTION}

In this section, we present a deep-learning-based method designed for PPG peak detection. The method is trained using noisy PPG signals. In the following, we first describe the data preparation step, including a generator function to produce noisy PPG signals. We then present the proposed model architecture and peak extraction method. The data analysis pipeline is shown in Figure 3

\section{A. DATA PREPARATION}

Data preparation generates noisy PPG signals with different SNRs to train and test the proposed model. The signals are generated using the available database, presented in Section IV] In this regard, we first extract clean PPG signals and noise from the database. The clean PPG signals are obtained using a PPG quality assessment technique, including five morphological features (i.e., spectral entropy, Shannon entropy, approximate entropy, kurtosis, and skewness) [33] and a Support Vector Machine method. We also obtain the systolic peak locations using a derivative base method [33]. Moreover, baseline wander and motion artifacts are extracted. Then, the clean signals (along with the peak locations) and noises are fed to a generator function.

\section{1) Generator Function}

A generator function is designed to create noisy PPG signals by aggregating clean PPG with noise. The noisy PPG signals are then utilized for training and testing the model. The generator function returns batches of normalized noisy PPG
Model

Developement

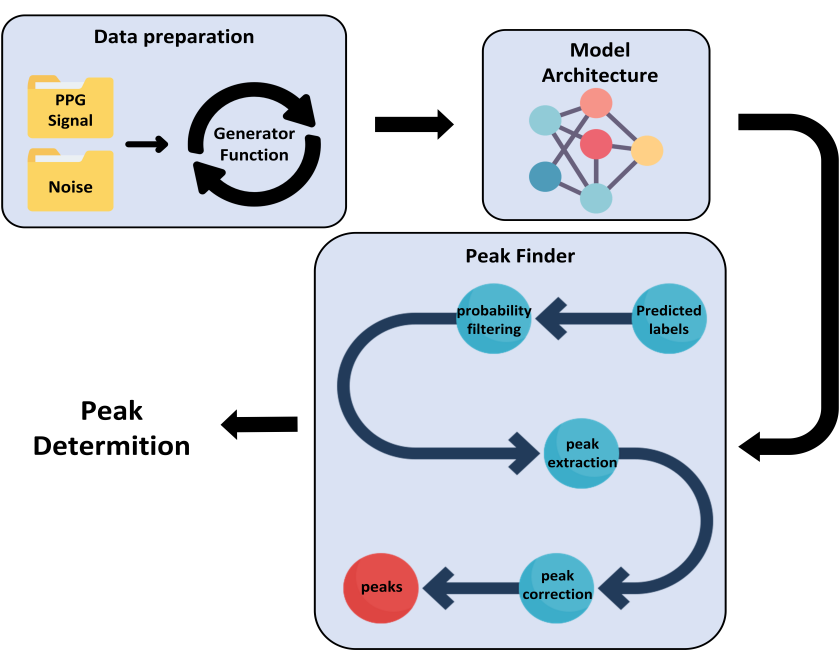

FIGURE 3. The proposed PPG peak detection method including data preparation, model architecture, and peak finder.

signals, their SNR values, and systolic peaks labels. Figure 4 a) shows a view of a generated PPG signal and its labeling vector.

The generator function includes 5 steps as follows:

Clean PPG signal selection: A 15-second window of clean PPG signal $(X)$ is randomly selected.

Noise selection: A 15 -second window of noise $(N)$ is randomly selected. Note that our dataset noise includes baseline wander and motion artifacts.

Noisy PPG generation: A weighted arithmetic mean is utilized to create the noisy PPG signals:

$$
S=w_{X} X+w_{N} N
$$

where $w_{X}$ and $w_{N}$ are the weights of the clean PPG signal and noise, respectively. In our case, $w_{X}$ is 1 while $w_{N}$ is a random number with uniform distribution $(0,5)$. Therefore,

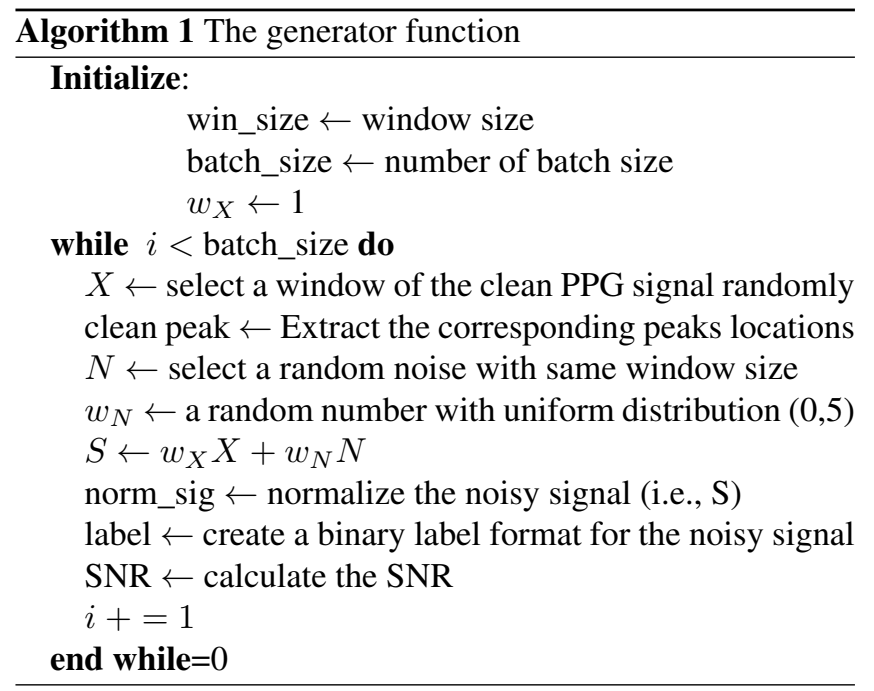


PPG signals with different noise levels are constructed. Then, the signals are normalized to $[-1,1]$ to be used for training and testing our model.

Labels extraction: A binary format is used for labeling the systolic peaks in the constructed PPG signal. In this labeling, " 1 " corresponds to the peak locations, whereas the rest of the signal is labeled as "0." Moreover, a slightly balanced "1" is added to the adjacent systolic peak points for making the model more robust against the false positive. In other words, despite considering one point as the location of the peaks, five labels (i.e., peak, two preceding and two succeeding points) are set to "1" (see Figure 4). The use of five "1" instead of only one "1" in the labeling vector leads to more robust positive predictions. Therefore, it reduces the noise effect in identifying the peak's location. It should be noted that the label values are created according to the systolic peaks in the clean PPG signals (but not in the aggregated noisy PPG).

SNR extraction: The SNR is calculated for each constructed

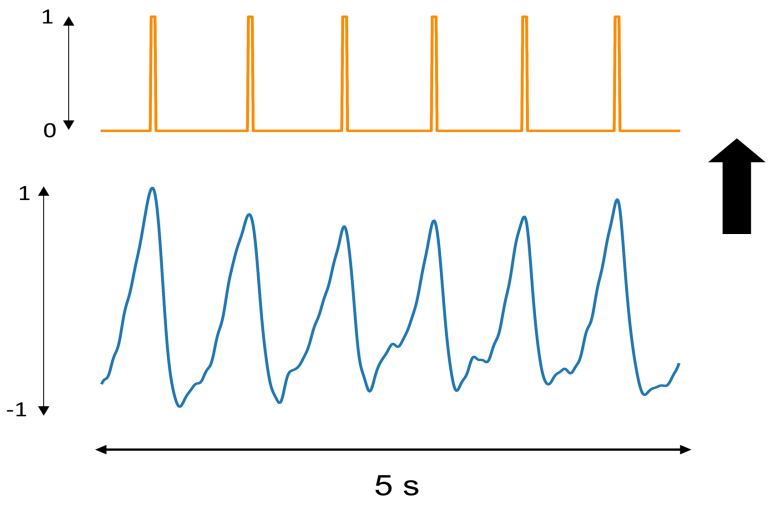

(a)

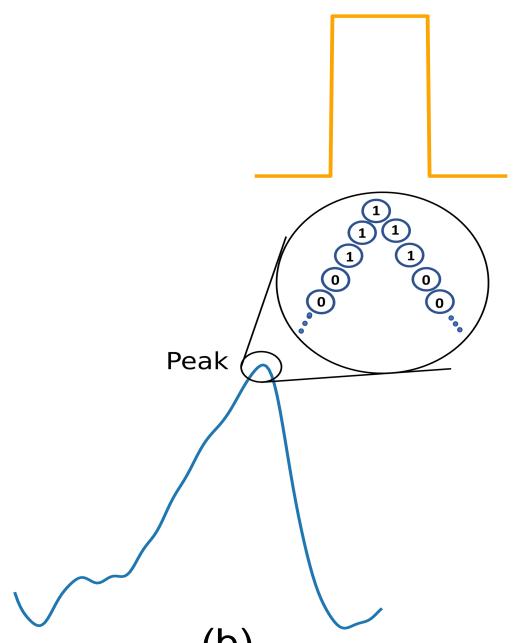

(b)

FIGURE 4. Schematic of labeling for PPG signal (a) a five-second PPG signal and its labeling vector. "1" indicates the peak's location (b) Five labels are set to " 1 " for each peak.

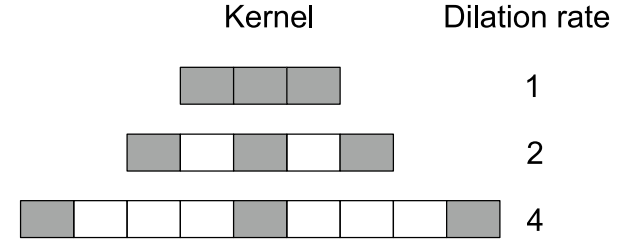

FIGURE 5. Receptive field of the kernel increases when dilation rate is increased. Grey denotes kernel weights while white indicates skipped inputs.

noisy PPG signal as follows:

$$
S N R=10 \log \frac{P_{\text {Signal }}}{P_{\text {Noise }}}
$$

where $P_{\text {Signal }}$ and $P_{\text {Noise }}$ are the signal and noise powers, respectively. The procedure of generator function is also indicated in Algorithm 1

\section{B. MODEL ARCHITECTURE}

To detect PPG peaks, we develop a CNN architecture with dilated convolutions, also known as atrous convolutions (or convolution with holes). Using dilated convolution instead of the regular one will result in a larger receptive field with the same amount of trainable parameters. This is achieved by inserting holes into the filter, i.e., some of the inputs are skipped as indicated in Figure 5. Dilation rate controls the amount of skipping, and filters with higher dilation rates have more holes. Dilated convolution with a dilation rate of 1 is a particular case that equals to a standard convolution. Dilated convolutions were utilized first time in efficient wavelet decomposition [34]. Later, they have been successfully utilized in different deep learning applications, such as semantic image segmentation [35], [36] and audio generation [37].

In our CNN model architecture, dilated convolutional layers are stacked, and their dilation rate is doubled at every layer. This approach results in vast receptive fields even with few layers (see Figure 6), which is computationally very efficient. Moreover, the input resolution is retained through the network. In contrast with our method, other existing methods that expand the receptive field like strided convolutions (stride larger than 1) or pooling layers reduce the spatial resolution [38]. Stacking dilated causal convolutional layers and simultaneously increasing dilation rate was first proposed by Oord et al. [37] in part of their wavenet architecture for audio generation. Our model is architecturally simpler, as we use a feedforward structure without any residual or skip connections. We also do not enforce causality. Therefore, receptive fields of the neurons in our model can contain both preceding and succeeding information, which will allow our model to make more accurate predictions.

Our model is fully convolutional, and it is a stack of 7 1D convolutional layers as indicated in Figure 7 The input resolution of 1500 time steps is retained through the model. The kernel size is also 3 for every layer. The model makes sequence to sequence mapping. It produces a probability value for every time step. The probability value indicates how 


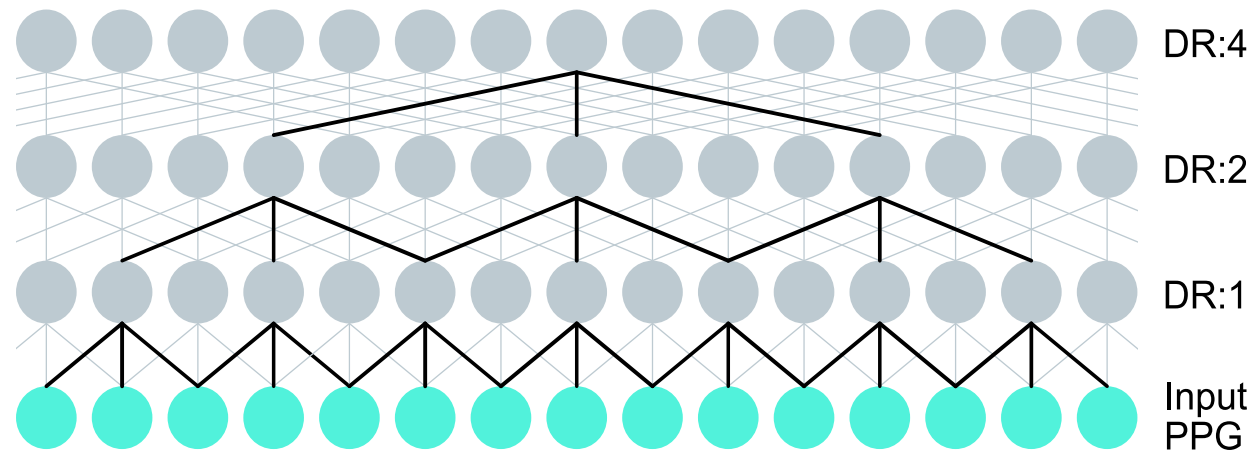

FIGURE 6. The receptive field of a neuron in a three-layer dilated convolution network is illustrated with bold lines. Note how the dilation rate (DR) is doubled at every layer. Our model is deeper than this illustration as it contains four additional layers.

\begin{tabular}{|l|}
\hline \multicolumn{1}{c}{ Input PPG } \\
\hline Filters: 4 , DR: 1 \\
\hline Filters: $8, \quad$ DR: 2 \\
\hline Filters: $8, \quad$ DR: 4 \\
\hline Filters: 16, DR: 8 \\
\hline Filters: 16, DR: 16 \\
\hline Filters: 32, DR: 32 \\
\hline Filters: $1, \quad$ DR: 64 \\
\hline
\end{tabular}

FIGURE 7. Our model is a fully convolutional neural network with seven layers. Dilatation rate (DR) is doubled at every layer while the number of filters is slowly increased with depth.

likely a signal point is a systolic peak. Two PPG examples with the probability values (i.e., the CNN model predictions) are shown in Figure 8. The dilatation rate is 1 in the first convolutional layer, and it is doubled at every following layer, reaching 64 at the final convolutional layer. This network structure results to a wide receptive field of 255 time steps for a neuron in a final classification layer. To keep our model compact, we slowly increase the number of filters as the network gets deeper. The first convolutional layer contains just four filters, while the second to last convolutional layer contains 32 filters. The final convolutional layer does the binary classification; therefore, it has only one filter. It uses sigmoid as an activation function while all preceding layers use exponential linear unit [39] as activation function. Moreover, we chose Adam [40] as an optimizer and binary crossentropy as loss function. The proposed model is very small since it only has 3169 trainable parameters.

\section{PEAK FINDER}

We develop a Wrapper function to extract the locations of the precise peaks from the model predictions, provided by the CNN model. Moreover, the Wrapper function detects and removes false peaks from the model predictions. Mainly, the
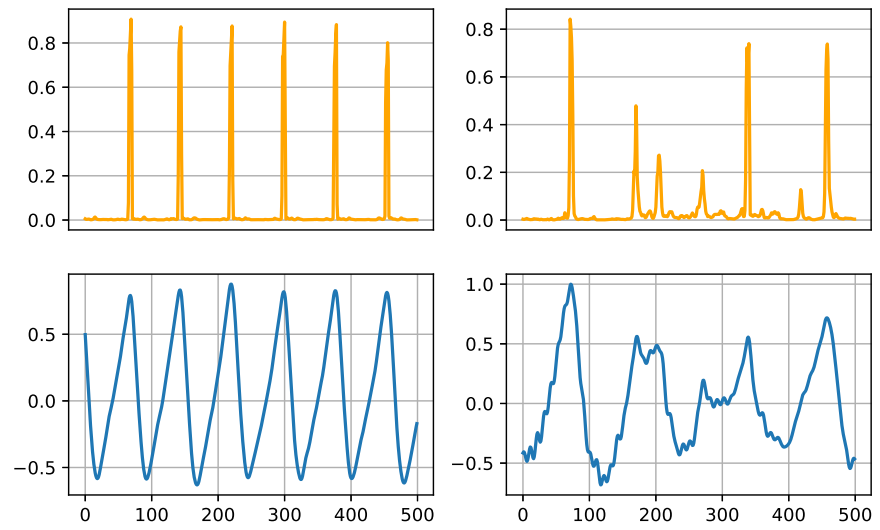

FIGURE 8. Two examples of inputs (i.e., PPG with different noise levels) and the model predictions. The lower row shows the two inputs, and the upper row includes the two labeling vectors predicted by model.

function performs the three following tasks:

1) Removing the peaks with low probability predictions.

2) Extracting the precise peak locations within the predicted values.

3) Discarding false peaks in the predictions.

In the following, we describe the three tasks of the Wrapper function in more details.

\section{1) Low probability signal removal}

In the first step, predictions with low probability are discarded using a threshold value. A labeling vector -which each time step indicates one probability value between 0 and $1-$ is fed to the wrapper function. Then, a local threshold filter is applied to the predicted time steps, and the time steps below the defined probability value are filtered out. The threshold is chosen empirically after a considerable number of predicted time steps evaluation. Decreasing the probability threshold improves the recall but reduces the precision.

\section{2) Peak Extraction}

We use a local maximum finder to determine the exact peak's location. For this purpose, we design a searching function to find the five samples segment that has the higher value 


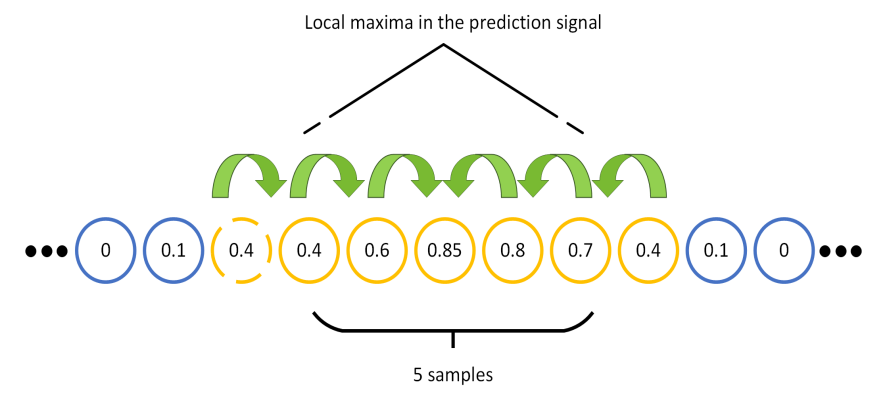

FIGURE 9. The procedure of selecting prediction that are above given threshold.

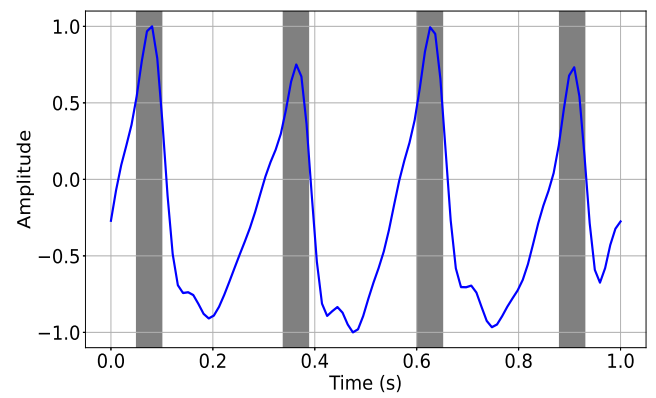

FIGURE 10. Tolerance distance $(50 \mathrm{~ms})$ is shaded in the figure. If the peak is detected within this range it is considered as true peak.

of the probability within the model predictions. In each five samples segment of the predicted time steps, the index of the higher probability is chosen as the location of the peak. Moreover, if there are two same probability values in the selected segment, the first probability value is chosen, and the corresponding index is extracted as the location of the peak. Figure 9 illustrates a segment of model prediction with its corresponding probability values. As shown in Figure 9 seven samples are above the threshold. In this example, the function finds the sample with the highest probability (i.e., $0.85)$ by comparing the neighbor points.

We produce a balance labeling vector for the noisy PPG signals, as instructed in Section $\mathrm{V}$. This idea helps our model to achieve higher precision while maintaining a lower tolerance distance. In other words, in the data generation stage, we introduce a new method for labeling. The method was generating a series of five "1" instead of only one "1" as the location of the peak. This means that if the algorithm finds a peak in the peak detection phase, there might be a time difference between the exact peak location and the detected peak. This time difference is introduced as tolerance distance. Figure 10 shows a segment of PPG signal and the defined tolerance distance with gray shaded rectangulars. In the proposed method, the tolerance distance is $50 \mathrm{~ms}$, which is smaller than the tolerance distance (i.e., $88 \mathrm{~ms}$ ) in other studies in the literature [41], [42].

\section{3) Peak correction}

In the third step, too-close peaks are discarded. Ventricular depolarization cannot occur in the refractory period despite the presence of stimuli. Therefore, no peak is presented in PPG signals during the refractory period after a peak. Our analysis assumes that the maximum heart rate is 200 beats per minute, and accordingly, the minimum distance between two successive peaks is 300 milliseconds. This step is necessary when we aim to maximize the recall while a low-value probability threshold is defined.

Accordingly, the PPG peaks within a distance less than the threshold (i.e., $300 \mathrm{~ms}$ ) are considered as false peaks. In this regard, we add a peak into the false-peak list if the distance with its preceding peak is less than $300 \mathrm{~ms}$. Then, the falsepeak list is sorted based on the peaks probabilities. In the next step, we select the highest peaks probability in the falsepeak list and add it to the peak list. Then, we calculate the distance with its preceding peak. If the distance would be larger than the threshold, it is chosen as a peak; otherwise, it is removed. We repeat this step until the false-peak list is empty. For clarity, let us take an example of the PPG peak correction. Four systolic peaks are indicated in Figure 11 In the first round, we calculate the distance between each peak with its preceding peak. As shown in the figure, the distance between the first peak $\left(P_{1}\right)$ and the second peak $\left(P_{2}\right)$ is $250 \mathrm{~ms}$. Therefore, $P_{2}$ is added to the false-peak list. Likewise, $P_{3}$ is added to the false-peak list. In the next step, we sort these false peaks based on their probabilities. Then, we start with the highest probability (i.e. $P_{2}$ ) and calculated its distance with $P_{1}$. The distance is $350 \mathrm{~ms}$, which is above the threshold. Hence, $P_{3}$ is considered as a systolic peak. In the next round, we choose $P_{2}$ and follow the same procedure. As the distance between $P_{2}$ and $P_{1}$ is less than the threshold, $P_{2}$ is not a systolic peak and is removed from the false-peak list.

\section{EVALUATION AND RESULTS}

We evaluate the proposed method in terms of accuracy using the PPG data collected via the Samsung smartwatches in free-living conditions. The evaluation includes the data of 36 healthy individuals. We validate the performance of the proposed method by implementing an inter-patient test, in which training and testing data are selected from separate individuals. In this regard, the PPG data of 26 participants (i.e., 9600000 15-second segments) are utilized for the training phase. We train the proposed model using 1) the noisy PPG signals constructed via the generator function and 2) their true labeling vectors. For the testing phase, the data of 10 participants (i.e., 3580015 -second segments) are selected. Similar to the training phase, the generator function is utilized to create noisy PPG segments. The test PPG signals are fed to the model, and the labeling vectors are estimated. Then, the method's performance is assessed by comparing the estimated labeling vectors with the true labeling vectors.

In our experiments, we used a Linux machine with AMD Ryzen Threadripper 2920X 12-Core processor, NVIDIA TITAN RTX GPU (24 GB memory), and 126 GB RAM. We use Tensorflow (v2) deep learning framework with high-level Keras API to construct our model. A batch size of 800 and 


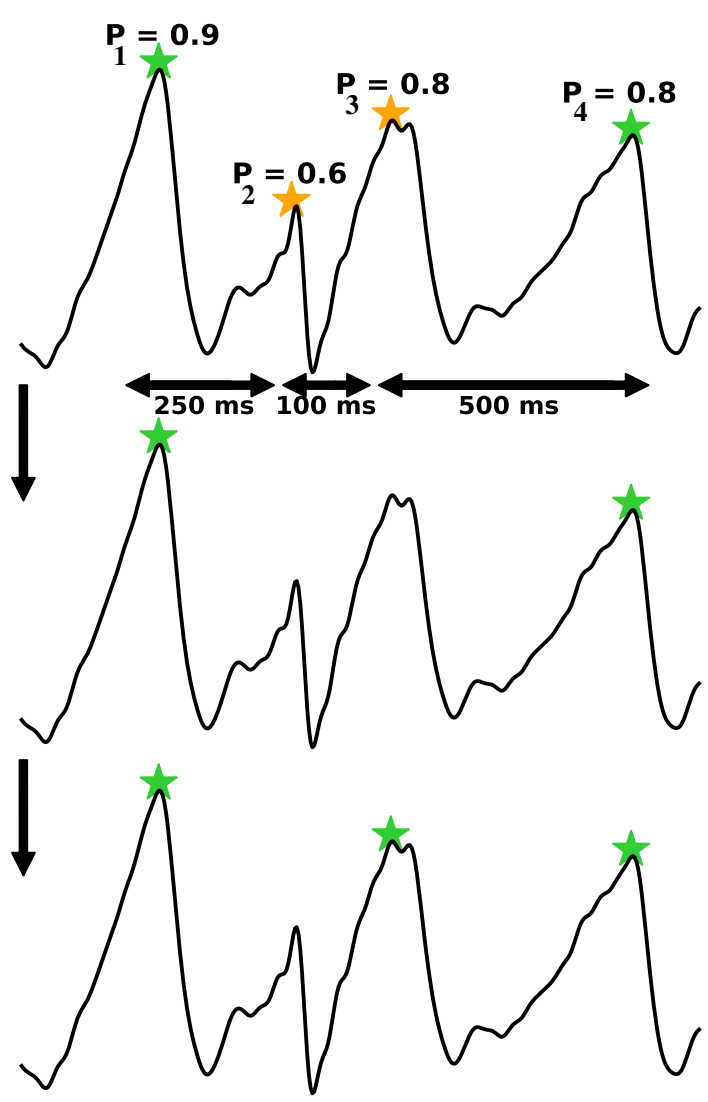

FIGURE 11. Filtering the unnaturally close peaks in the peak correction step. The upper figure shows all of the detected peaks. The middle plot illustrates the removal of the peaks that are within the threshold distance. The lowermost figure shows the peak with the highest probability is added back to the final set of accepted peaks.

200 epochs, where the number of steps per epoch was 60 , was selected for model training. In the training data, the range of SNR is from -2.5 to $47.5 \mathrm{~dB}$ (complete noisy to noise-free signal). The data are clustered into 10 ranges with the step of $5 \mathrm{~dB}$. This balancing prevents the network from being overlearned for a specific SNR value. A total number of 9600000 segments were used for the training phase $(90 \%$ training and $10 \%$ validation). Figure 12 indicated four examples of 15seconds noisy PPG signals, with different noise levels, used in the training phase. The method was implemented using Tensorflow [43], Keras [44], and SciPy [45] in Python.

In addition to the proposed method, we implement four exiting methods for PPG peak detection. First, Elgendi et al. method [46] is performed, in which a dynamic threshold and two event-related moving average methods are utilized. Second, we utilize Van Gent et al. method [26] as an adaptive threshold method. Van Gent et al. [26] uses an adaptive threshold along with a moving average on both sides of each sample. Third, Kuntamalla et al. [8] method is implemented to estimate PPG peaks using an adaptive threshold, which is empirically set to 0.35 . Fourth, Chakraborty et al. [29] as a transform-based method is used to estimate the peaks'
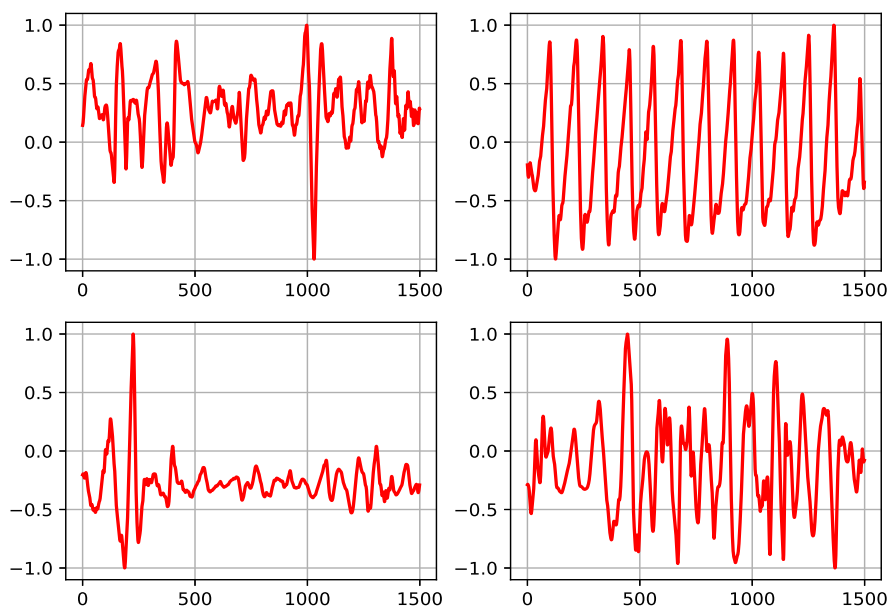

FIGURE 12. Examples of PPG data with different noise levels used in the training phase. The upper right figure illustrates a low noise PPG signal, while the other examples contain different types of noises such as motion artifact and baseline wander.

locations using a Hilbert transform. It should be noted that For the Elgendi and Van Gent methods, we use the versions that are implemented in Neurokit [47] and Heartpy [48] Python packages.

\section{A. EVALUATION MEASURES}

A beat-to-beat comparison was made between the detection results and the reference test set label to evaluate the algorithm in terms of accuracy. In the comparison, true-positive (TP) is when the PPG peaks are detected correctly, falsenegative (FN) is when the method fails to detect a peak, and false-positive (FP) is when the algorithm detects, e.g., noise as a peak. Then, the performance of the proposed method is assessed by calculating precision, recall, and F1-score as follows [28]:

$$
\begin{gathered}
\text { precision }=\frac{\mathrm{TP}}{\mathrm{TP}+\mathrm{FP}} \\
\text { recall }=\frac{\mathrm{TP}}{\mathrm{TP}+\mathrm{FN}} \\
\mathrm{F} 1 \text {-score }=2 \times \frac{\text { precision } \times \text { recall }}{\text { precision }+ \text { recall }} \\
=2 \times \frac{\mathrm{TP}}{\mathrm{TP}+\mathrm{FP}+\mathrm{FN}}
\end{gathered}
$$

\section{B. TEST SET RESULTS}

Our proposed method is evaluated using the test dataset created by the generator function. The function generates $100 \mathrm{~Hz}$ noisy PPG signals along with the SNR values and the corresponding labeling vectors. 35800 noisy PPG signals with a balanced range of SNR are used for the testing. The SNR values are between -2.5 and $47.5 \mathrm{~dB}$ in our evaluation. The signals are divided into 5-dB-SNR groups. Then, the performance of the methods is investigated for each group. 

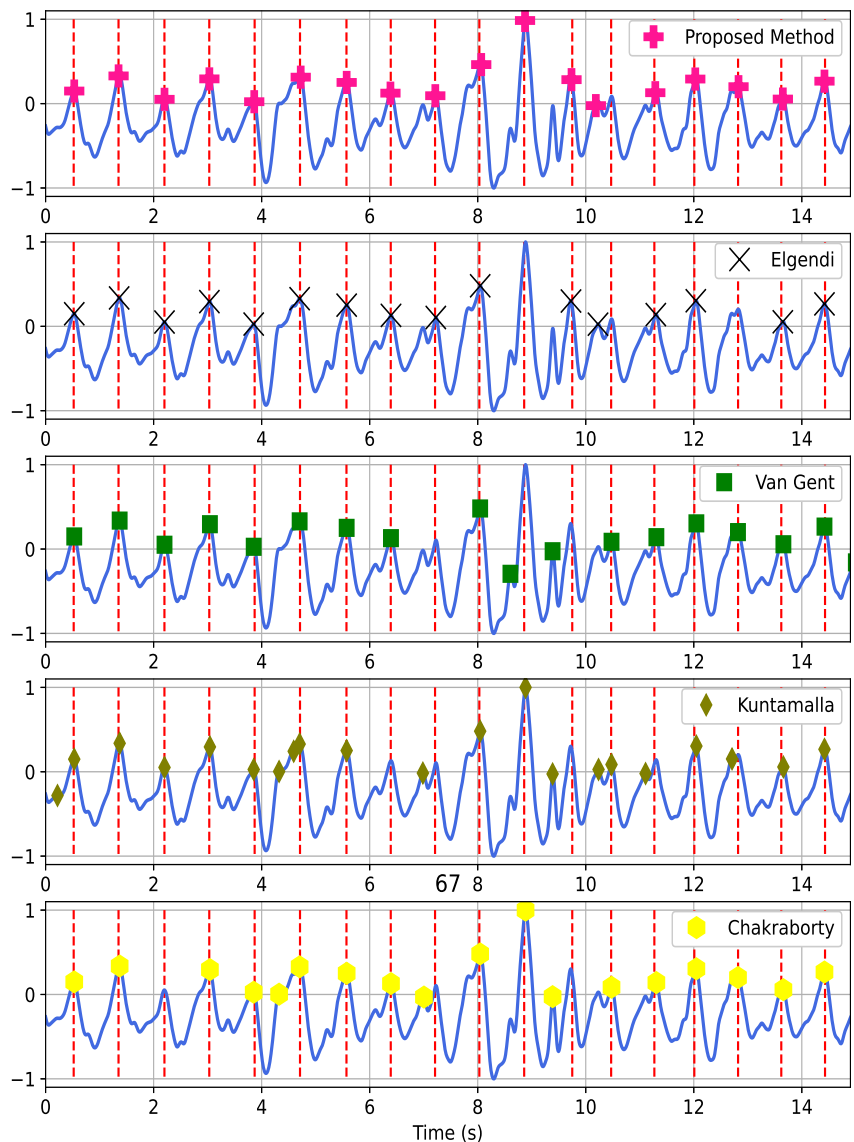

FIGURE 13. A PPG signal segment with $6.82 \mathrm{~dB}$ SNR and the peak detection results obtained by the methods. The vertical dash lines are true peak. The markers show the positions of the peaks detected by the methods.

Figure 13 shows a PPG segment with different peak detection results. The SNR is $6.82 \mathrm{~dB}$. The vertical dash lines show the true peaks, and the markers indicates the estimated peaks. Our method misses one systolic peak in second 10.8. However, the other methods miss several peaks and detect false peaks as systolic peaks. The Kuntamalla method had the worst performance in this example.

The performance of the models for different SNR groups are shown in Figure 14. A quantitative comparison is also presented in Table 2 Figure 14(a) illustrates the methods' precision. All the methods except the Kuntamalla method obtain equal precision value (i.e., 97\%) when the SNR is above $42.5 \mathrm{~dB}$. However, the precision values drop when the SNR values decrease. For example, in SNR 45 to $25 \mathrm{~dB}$, the precision for the proposed method, Elgendi, Van Gent, Chakraborty, and Kuntamalla decreased by $15 \%, 18 \%, 24 \%$, $19 \%$, and $20 \%$, respectively. As indicated, the proposed deep learning-based method outperforms the existing methods. The results show that the false positive in the proposed method is lower compared to the other methods. Therefore, our method detects fewer false peaks as systolic peaks in the noisy PPG signals.

Figure 14(b) indicates the methods' Recall values. The

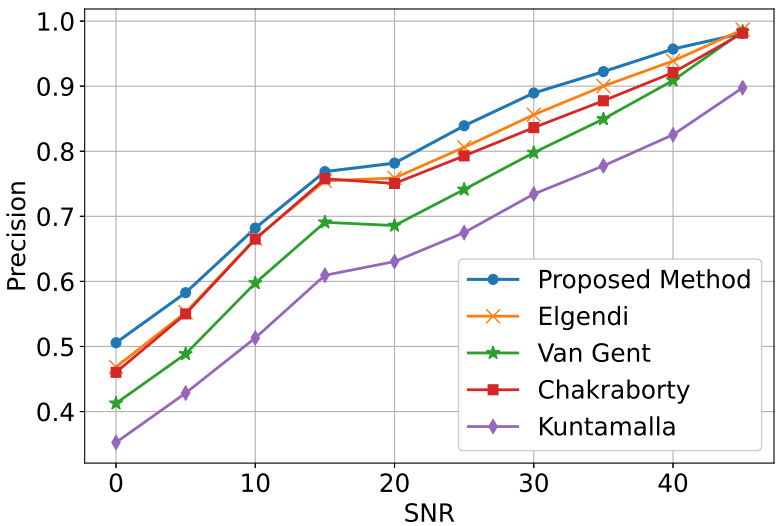

(a)

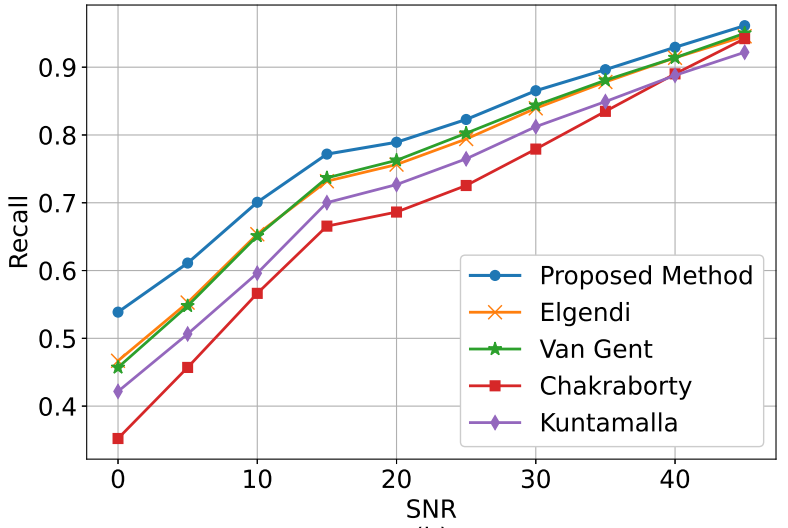

(b)

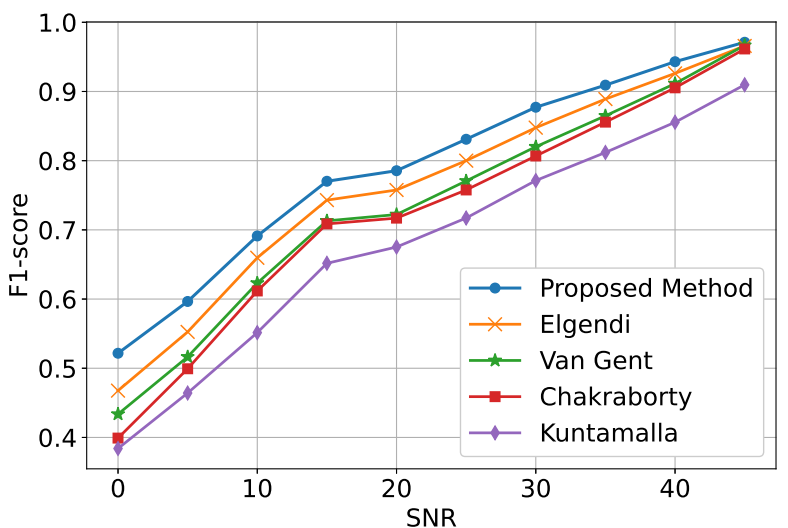

(c)

FIGURE 14. Performance comparison between the methods. (a) precision (b) recall (c) F1-score at different noise levels.

figure shows that all the methods perform well in noisefree conditions, i.e., almost $96 \%$ recall. As indicated, there are decreasing trends in the recall values when the SNR decreases. The falling trends are more intense in lower SNRs. With the least SNR, the difference between our method and the other methods reaches the highest value. As presented in Table 2 in SNR $0 \mathrm{~dB}$, the differences between our method and the other methods are 7\% (Elgendi), 8\% (Van Gent), $12 \%$ ( Kuntamalla), and $18 \%$ (Chakraborty). Similar to the precision, our method performs better in all SNR groups compared to the existing methods. The recall values show 
TABLE 2. Performance comparison between the proposed method and the other existing methods

\begin{tabular}{|c|c|c|c|c|c|c|c|c|c|c|c|c|c|c|c|}
\hline \multirow{2}{*}{$\begin{array}{l}\text { SNR } \\
(\mathrm{dB})\end{array}$} & \multicolumn{3}{|c|}{ Proposed method } & \multicolumn{3}{|c|}{ Elgendi } & \multicolumn{3}{|c|}{ Van Gent } & \multicolumn{3}{|c|}{ Chakraborty } & \multicolumn{3}{|c|}{ Kuntamalla } \\
\hline & prec. & recall & $\mathrm{F} 1$ & prec. & recall & $\mathrm{F} 1$ & prec. & recall & $\mathrm{F} 1$ & prec. & recall & $\mathrm{F} 1$ & prec. & recall & $\mathrm{F} 1$ \\
\hline 45 & 0.98 & 0.96 & 0.97 & 0.98 & 0.94 & 0.96 & 0.98 & 0.95 & 0.96 & 0.98 & 0.94 & 0.96 & 0.89 & 0.92 & 0.90 \\
\hline 40 & 0.95 & 0.92 & 0.94 & 0.93 & 0.90 & 0.92 & 0.91 & 0.91 & 0.91 & 0.92 & 0.88 & 0.90 & 0.82 & 0.88 & 0.85 \\
\hline 35 & 0.93 & 0.89 & 0.91 & 0.90 & 0.88 & 0.89 & 0.86 & 0.88 & 0.87 & 0.88 & 0.83 & 0.85 & 0.78 & 0.85 & 0.81 \\
\hline 30 & 0.89 & 0.87 & 0.88 & 0.87 & 0.85 & 0.86 & 0.81 & 0.85 & 0.83 & 0.85 & 0.79 & 0.82 & 0.74 & 0.82 & 0.78 \\
\hline 25 & 0.83 & 0.82 & 0.83 & 0.80 & 0.79 & 0.80 & 0.74 & 0.80 & 0.77 & 0.79 & 0.73 & 0.76 & 0.69 & 0.77 & 0.73 \\
\hline 20 & 0.78 & 0.78 & 0.78 & 0.76 & 0.75 & 0.76 & 0.69 & 0.76 & 0.72 & 0.75 & 0.68 & 0.71 & 0.63 & 0.73 & 0.67 \\
\hline 15 & 0.76 & 0.77 & 0.76 & 0.75 & 0.73 & 0.74 & 0.68 & 0.73 & 0.71 & 0.75 & 0.66 & 0.70 & 0.60 & 0.69 & 0.64 \\
\hline 10 & 0.68 & 0.70 & 0.69 & 0.66 & 0.66 & 0.66 & 0.60 & 0.66 & 0.63 & 0.67 & 0.58 & 0.62 & 0.52 & 0.61 & 0.56 \\
\hline 5 & 0.58 & 0.62 & 0.60 & 0.55 & 0.56 & 0.56 & 0.49 & 0.55 & 0.52 & 0.55 & 0.46 & 0.50 & 0.42 & 0.50 & 0.46 \\
\hline 0 & 0.50 & 0.53 & 0.52 & 0.46 & 0.46 & 0.46 & 0.42 & 0.45 & 0.43 & 0.46 & 0.35 & 0.40 & 0.35 & 0.41 & 0.38 \\
\hline Overall & 0.80 & 0.80 & 0.80 & 0.78 & 0.76 & 0.77 & 0.72 & 0.77 & 0.74 & 0.78 & 0.76 & 0.77 & 0.65 & 0.73 & 0.69 \\
\hline
\end{tabular}

* The corresponding PPG records with the highest precision, recall, or F1 scores (in each row) are presented in bold type.

* The number of signals analysed for each SNR range are 3580

that our method obtains lower false negatives. Therefore, our method is more successful in detecting the true peaks.

Finally, the methods' F1-score values are illustrated In Figure 14(c). When the SNR values drop, the F1-score of the proposed method decrease with a smaller slope compared to the existing methods. The difference is bigger with lower SNR values. For example, as shown in Table 2, the F1-score values in $0 \mathrm{~dB}$ SNR are 0.52, 0.46, 0.43, 0.40, and 0.38 for our method, Elgendi, Van Gent, Chakraborty, and Kuntamalla, respectively. Consequently, the proposed method has the best performance with all the SNR groups, particularly when the SNR values are small. The method is more robust against noises and could better discriminate between the systolic and noise peaks.

\section{LIMITATIONS AND FUTURE WORK}

The dataset used in this paper was limited to healthy participants. However, other studies [30] indicated that arrhythmias -such as premature atrial contraction, premature ventricle contraction, and atrial fibrillation- might affect the accuracy of peak detection methods. The method's performance should be investigated with the data of non-healthy individuals to address the lack of generalizability of the results.

Moreover, our evaluation was restricted to one dataset collected during free-living conditions using Samsung Gear Sport smartwatches. The method performance should be evaluated with different physical activities. In our future work, we intend to validate our method with other databases, such as [49], [50], in which the users are engaged more in intense physical activities such as cycling and running.

\section{CONCLUSION}

In this paper, we presented a robust CNN-based peak detection for PPG signals with different noise levels. The proposed method included three phases.

A generator function was introduced in the first phase, combining the PPG records with different noise levels. In the second phase, a dilated CNN was proposed. The use of dilated convolutions provided a large receptive field, which enhanced the efficiency of time series processing with CNNs. In the third phase, a wrapper function was implemented to detect the location of the PPG signals. After predicting the peaks, a filtering function was used to remove the false peaks. We evaluate the proposed method using the PPG data collected via wearable devices under free-living conditions. Our method was compared with 4 existing PPG peak detection methods. The performance of the methods were similar with noise-free PPG. However, our method exhibited higher accuracy when the noise level increased. We showed that the average F1-score of the proposed method was $80 \%$, while Elgendi, Van Gent, Chakraborty, and Kuntamalla methods obtained $77 \%, 74 \%, 77 \%$, and $69 \%$, respectively. Our results indicated that the proposed PPG peak detection method was more successful in terms of recall and precision in a noisy environment.

\section{ACKNOWLEDGMENT}

The authors would like to thank Fatemeh Sarhaddi, Anna Axelin, Hannakaisa Niela-Vilen, Elisa Lankinen, Mohsen Saei Dehghan, Bushra Zafar, and Henrika Merenlehto for contributing to the data collection. 


\section{References}

[1] F. Firouzi et al., "Internet-of-Things and big data for smarter healthcare: From device to architecture, applications and analytics," Future Generat. Comput. Syst., vol. 78, pp. 583-586, Jan. 2018.

[2] Mieronkoski et al., "The Internet of Things for basic nursing careA scoping review", Int. J. Nursing Stud., vol. 69, pp. 78-90, Apr. 2017, [online] Available: https://doi.org/10.1016/j.ijnurstu.2017.01.009.

[3] J. Allen, "Photoplethysmography and its application in clinical physiological measurement", Physiol. Meas., vol. 28, pp. R1-R39, 2007.

[4] S. Majumder, T. Mondal and M. J. Deen, "Wearable sensors for remote health monitoring", Sensors, vol. 17, no. 1, pp. 130, 2017.

[5] MIT Laboratory for Computational Physiology. Physionet Databases. 2021 [Online]. Available: https://physionet.org/about/database/.

[6] D. G. Jang, U. Farooq, J. H. Park, S. H. Park and M. Hahn, "An adaptive SSF-based pulse peak detection algorithm for heart rate variability analysis in home healthcare environments", Proc. 7th Int. Conf. Ubiquitous Healthcare, pp. 70-71, 2010.

[7] N. Paradkar and S. R. Chowdhury, "Primary study for detection of arterial blood pressure waveform components", Proc. 37th Int. Conf. IEEE EMBS, pp. 1959-1962, Aug. 2015.

[8] S. Kuntamalla and L. R. G. Reddy, "An Efficient and Automatic Systolic Peak Detection Algorithm for Photoplethysmographic Signals", Int. J Comput. Appl., vol. 97, no. 19, 2014.

[9] V. Ostojić, T. Lončar-Turukalo and D. Bajić, "Empirical mode decomposition based real-time blood pressure delineation and quality assessment", Proc. IEEE Comput. Cardiol., vol. 40, pp. 221-224, Sep. 2013.

[10] D.-G. Jang, U. Farooq, S.-H. Park and M. Hahn, "A robust method for pulse peak determination in a digital volume pulse waveform with a wandering baseline", IEEE Trans. Biomed. Circuits Syst., vol. 8, no. 5, pp. 729-737, Oct. 2014.

[11] D. G. Jang, S. Park, M. Hahn and S. H. Park, "A real-time pulse peak detection algorithm for the photoplethysmogram", Int. J. Electron. Electr. Engin., vol. 2, pp. 45-49, 2014.

[12] T. Bhowmik, J. Dey and V. N. Tiwari, "A novel method for accurate estimation of HRV from smartwatch PPG signals", Proc. 39th Annu. Int. Conf. IEEE Eng. Med. Biol. Soc. (EMBC), pp. 109-112, Jul. 2017.

[13] B. R. Ferro, A. R. Aguilera and R. R. Fernandez De La Vara Prieto, "Automated detection of the onset and systolic peak in the pulse wave using Hilbert transform", Biomedical Signal Processing and Control, vol. 20, pp. 78-84, Jul. 2015.

[14] M. S. Manikandan and K. P. Soman, "A novel method for detecting R-peaks in electrocardiogram (ECG) signal", Biomed. Signal Process. Control, vol. 7, no. 2, pp. 118-128, Mar. 2012.

[15] A. Anzanpour, D. Amiri, I. Azimi, M. Levorato, N. Dutt, P. Liljeberg, A.M Rahmani, "Edge-Assisted Control for Healthcare Internet of Things: A Case Study on PPG-Based Early Warning Score". ACM Trans. Internet Things vol. 2, no. 1, pp. 1-21, 2020.

[16] E. K. Naeini, I. Azimi, A. M. Rahmani, P. Liljeberg and N. Dutt, "A realtime PPG quality assessment approach for healthcare internet-of-things", Procedia Computer Science, vol. 151, pp. 551-558, 2019.

[17] C. C. Aggarwal, "Neural Network and Deep Learning", Springer, 2018.

[18] A.D. Orjuela-Cañón, D. Delisle-Rodríguez, A. López-Delis, R.F. de la Vara-Prieto, M.B. Cuadra-Sanz "Onset and peak pattern recognition on photoplethysmographic signals using neural networks", 18th Iberoamerican Congress on Progress in Pattern Recognition, Image Analysis, Computer Vision, and Applications, Springer, 2013, Berlin, Garmany, pp. 543550

[19] A. Reiss, I. Indlekofer, P. Schmidt and K. Van Laerhoven, "Deep PPG: large-scale heart rate estimation with convolutional neural networks", Sensors, vol. 19, no. 14, pp. 3079, 2019.

[20] T. V. Tran and W.-Y. Chung, "A robust algorithm for real-time peak detection of photoplethysmograms using a personal computer mouse", IEEE Sensors J., vol. 15, no. 8, pp. 4651-4659, Aug. 2015.

[21] C. Fischer, B. Dömer, T. Wibmer and T. Penzel, "An algorithm for realtime pulse waveform segmentation and artifact detection in photoplethysmograms", IEEE J. Biomed. Health Inform., vol. 21, no. 2, pp. 372-381, Mar. 2017.

[22] I. Iliev, B. Nenova, I. Jekova and V. Krasteva, "Algorithm for real-time pulse wave detection dedicated to non-invasive pulse sensing", Proc. Comput. Cardiol. (CinC), pp. 777-780, Sep. 2012.

[23] R. R. F. de la Vara Prieto, D. D. Rodríguez, M. B. C. Sanz, A. S. Mengana and H. F. P. Quintero, "Algorithm for systolic peak detection of pulse wave", Proc. 38th Conf. Latinoamericana Inform. (CLEI), pp. 1-4, Oct. 2012 .
[24] S. Vadrevu and M. S. Manikandan, "Effective systolic peak detection algorithm using variational mode decomposition and center of gravity", 2016 IEEE Region 10 Conference (TENCON), pp. 2711-2715, 2016.

[25] H. S. Shin, C. Lee and M. Lee, "Adaptive threshold method for the peak detection of photoplethysmographic waveform", Computers in Biology and Medicine, vol. 39, no. 12, pp. 1145-1152, 2009.

[26] P. van Gent, H. Farah, N. van Nes and B. van Arem, "Analysing noisy driver physiology real-time using off-the-shelf sensors: Heart rate analysis software from the taking the fast lane project", J. Open Res. Softw., vol. 7 , pp. 1-9, 2019.

[27] P. van Gent, H. Farah, N. van Nes and B. van Arem, "Heartpy: A nove heart rate algorithm for the analysis of noisy signals", Transportation research part $F$ : traffic psychology and behaviour, vol. 66, pp. 368-378, 2019.

[28] S. Vadrevu and M. S. Manikandan, "A robust pulse onset and peak detection method for automated PPG signal analysis system", IEEE Trans. Instrum. Meas., vol. 68, no. 3, pp. 807-817, Mar. 2019.

[29] A. Chakraborty, D. Sadhukhan, M. Mitra, "A robust PPG onset and systolic peak detection algorithm based on Hilbert transform", In Proc. 2020 IEEE Calcutta Conf. (CALCON), Kolkata, India, 28-29 Feb. 2020.

[30] D. Han et al., "Smartwatch PPG peak detection method for sinus rhythm and cardiac arrhythmia", Proc. 41st Annu. Int. Conf. IEEE Eng. Medicine Biol. Soc., pp. 4310-4313, 2019.

[31] M. Asgari Mehrabadi et al. "Sleep tracking of commercially available smart ring and watch against medical-grade actigraphy in everyday settings: instrument validation study", JMIR Mhealth Uhealth, vol. 8, no. 10, 2020.

[32] Samsung Gear Sport. https://www.samsung.com/global/galaxy/gear-sport Accessed: 2010-09-30.

[33] A. Mahmoudzadeh, I. Azimi, A. M. Rahmani, P. Liljeberg, 'Lightweight Photoplethysmography Quality Assessment for Real-time IoT-based Health Monitoring using Unsupervised Anomaly Detection”, Procedia Computer Science, vol. 184, pp. 140-147, 2021.

[34] M. Holschneider, R. Kronland-Martinet, J. Morlet and P. Tchamitchian, "A real-time algorithm for signal analysis with the help of the wavelet transform", Proc. Wavelets: Time-Frequency Methods Phase Space, pp. 289-297, 1989.

[35] L.C. Chen, G. Papandreou, I. Kokkinos, K. Murphy and A. L. Yuille, "DeepLab: Semantic image segmentation with deep convolutional nets atrous convolution and fully connected CRFs", IEEE Trans. Pattern Anal. Mach. Intell., vol. 40, no. 4, pp. 834-848, 2017.

[36] L.-C. Chen, G. Papandreou, F. Schroff and H. Adam, "Rethinking Atrous convolution for semantic image segmentation", 2017, [online] Available: https://arxiv.org/abs/1706.05587.

[37] A. van den Oord, S. Dieleman, H. Zen, K. Simonyan, O. Vinyals, A Graves, N. Kalchbrenner, A. Senior, and K. Kavukcuoglu. "Wavenet: A generative model for raw audio", arXiv preprint arXiv:1609.03499, 2016.

[38] Z. Wang and S. Ji, "Smoothed dilated convolutions for improved dense prediction", Proceedings of the 24th ACM SIGKDD International Conference on Knowledge Discovery \& Data Mining, ACM, pp. 2486-2495 2018.

[39] D.-A. Clevert, T. Unterthiner and S. Hochreiter, Fast and accurate deep network learning by exponential linear units (ELUs), arXiv preprint, Available: https://arXiv.org/1511.07289, 2015.

[40] D. P. Kingma and J. L. Ba, "Adam: A method for stochastic optimization",arXiv preprint, Available: https://arXiv.org/1412.6980, 2014

[41] G. M. Friesen, "A comparison of the noise sensitivity of nine QRS detection algorithms", IEEE Trans. Biomed. Eng., vol. 37, pp. 85-98, Jan. 1990.

[42] J. Laitala et al., "Robust ECG R-peak detection using LSTM", Proc. ACM Symp. Appl. Comput., pp. 1104-1111, Mar. 2020.

[43] M. Abadi et al., "TensorFlow: Large-Scale Machine Learning on Heterogeneous Systems", 2015, [online] Available: http://tensorflow.org/.

[44] F. Chollet. "keras.", 2015, [Online]. Available: https://keras.io.

[45] P. Virtanen, R. Gommers, T. E. Oliphant, M. Haberland, T. Reddy, D. Cournapeau, et al., "SciPy I. 0-Fundamental Algorithms for Scientific Computing in Python", Jul 2019.

[46] M. Elgendi, I. Norton, M. Brearley, D. Abbott and D. Schuurmans, "Systolic peak detection in acceleration photoplethysmograms measured from emergency responders in tropical conditions", PLOS ONE, vol. 8, no. 10, pp. e76585, 2013.

[47] D. Makowski, et al., "NeuroKit2: A Python toolbox for neurophysiological signal processing Behavior Research Methods", 2021, Available:https://doi.org/10.3758/s13428-020-01516-y 
[48] P. van Gent, H. Farah, N. van Nes and B. van Arem, "Heart Rate Analysis for Human Factors: Development and Validation of an Open Source Toolkit for Noisy Naturalistic Heart Rate Data", Proc. 6th HUMANIST Conf., no. June, pp. 173-178, 2018.

[49] Z. Zhang, "IEEE Signal Processing Cup 2015: Heart Rate Monitoring During Physical Exercise Using Wrist-Type Photoplethysmographic (PPG) Signals", Accessed: Jun. 15, 2021.[Online]. Available: https://sites.google.com/site/researchbyzhang/ieeespcup2015

[50] D. Jarchi and A. J. Casson, "PhysioBank,PhysioToolkit, and PhysioNet: Components of a newresearch resource for complex physiologic signals", [Online]. Available: https://physionet.org/content/wrist/1.0.0/. 